\title{
Machine Learning for Software Engineering: a Bibliometric Analysis from 2015 to 2019
}

\author{
Ruben Heradio \\ Universidad Nacional de \\ Educacion a Distancia (UNED), \\ Madrid, Spain \\ rheradio@issi.uned.es
}

\begin{abstract}
The increase of computer processor speed and the ubiquitous availability of data coming from a diversity of sources (e.g., version control systems, software developers forums, operating system logs, etc.) have boosted the interest in applying machine learning to software engineering. Accordingly, the research literature on this topic has increased rapidly. This paper provides a comprehensive overview of that literature for the last five years. To do so, it examines 1,312 records gathered from Elsevier Scopus, identifying (i) the most productive authors and their collaboration networks, (ii) the countries and institutions that are leading research, (iii) the journals that are publishing the most papers, and (iv) the most important research themes and the highest impacted articles for those themes.
\end{abstract}

\section{Introduction}

The volume of data captured and stored from software applications, version control systems, software developers forums, operating system logs, application reviews, etc. grows incessantly. Machine Learning (ML) opens the possibility of processing that data for a variety of purposes.

For instance, the failures reported on existing software systems may be used for training a ML algorithm to predict potential failures in new systems according to some code features (e.g., cyclomatic complexity, lines of code, etc.) [1, 2, 3, 4]. Likewise, ML can be used to estimate software development cost/effort $[5,6]$, to trace dependencies between software artifacts [5, 6], to refactor code [7], to classify application reviews [8], to detect malware [9], etc.

According to Elsevier Scopus, the number of publications on ML for software engineering has grown from 110 papers in 2015 to 485 articles in 2019 (i.e., a $340.91 \%$ increment). This paper aims to provide a systematic analysis of the increasing large literature that is being generated about this topic. Thus, supporting the

\author{
Cristina Cerrada \\ UNED, \\ Madrid, Spain \\ ccerrada01@gmail.com
}

\author{
Manuel J. Cobo \\ University of Cadiz, \\ Cadiz, Spain \\ manueljesus.cobo@uca.es
}

identification of the research with the highest potential for industry and future investigations.

In particular, this paper targets the following Research Questions:

- RQ1: How fast is the number of publications growing?

- RQ2: Who are the most productive authors, and how do they collaborate?

- RQ3: What countries and institutions are leading research?

- RQ4: What journals have published most articles?

- RQ5: What are the most relevant themes of research, and what are the highest impacted papers for those themes?

To answer these questions, a sample of 1,312 articles retrieved from Elsevier Scopus is examined. Analyzing such a huge sample by hand would be difficult and error-prone. Alternative automated procedures are preferable $[10,11]$. Consequently, we have used two widespread bibliometric methods [12]: performance analysis and science mapping, which have been successfully applied in recent studies (e.g., [13, 14, 15]) since they complement each other thoroughly: performance analysis determines the significance of the bibliometric elements, and science mapping models how those elements are interrelated.

The remaining of this paper is organized as follows: Section 2 describes the materials and methods used to perform the analysis; Section 3 summarizes and discusses the most relevant results; finally, Section 4 provides some concluding remarks and points directions for future research.

\section{Materials and Methods}

This section describes the systematic methodology we have followed to gather and analyze a representative 
bibliometric corpus of articles dealing with the application of ML to software engineering from 2015 to 2019 .

\subsection{Bibliometric workflow}

According to the recommendations given by Cobo et al. [10], PRISMA [16], and Börner et al. [17], we followed a workflow composed of the following stages:

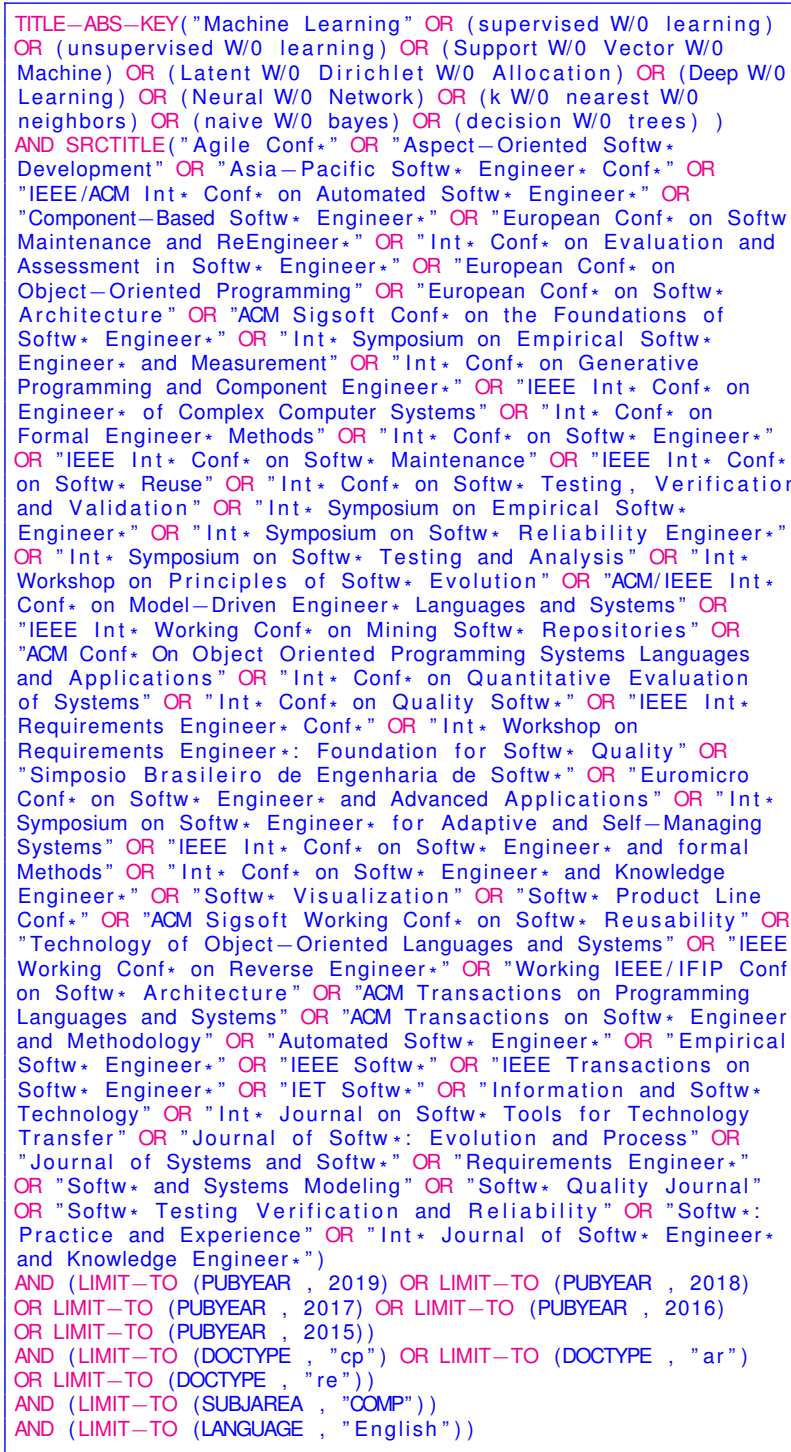

Figure 1: Query to gather the paper sample.

1. Data retrieval. Gathering the complete population of all research literature published about a given topic is usually unrealistic [18, 19]. In other words, exhaustiveness has to be substituted by representativeness. To obtain an unbiased publication sample that represents the population satisfactorily, the query in Figure 1 was executed on Elsevier Scopus, gathering 1,312 documents. Lines 1-5 specify the topic (i.e., machine learning); Lines 6-52 set the publication sources (40 conferences and 17 journals on software engineering); Lines 53-55 constraint the query to the period 2015-2019; Lines 56-57 limit the type of publication to conference paper ( $c p)$, journal article (ar), and review (re); Line 58 constraints the search to the computer science subject area; and Line 59 filters the results to documents written in English. It is worth noting that the query was iteratively polished until a good balance between completeness and absence of false-positives was reached.

2. Data normalization. Bibliographic data often includes typos and ambiguities [10, 11, 20]; e.g., an author may appear in several records with slightly changed names, or the same concept may correspond to different keywords. As these problems can bias the analysis, we standardized the bibliographic data manually.

3. Data analysis. The normalized data was examined using performance analysis (publication counts and citations) combined with a bibliometric technique called science mapping.

\subsection{Science mapping}

The Scimat tool ${ }^{1}$ was used to identify the most relevant topics and their role in the area by using two complementary techniques: thematic network clustering and strategic diagrams.

To recognize the most important research topics, the equivalence index [21] between each pair of keywords $i$ and $j$ was calculated as $e_{i j}=c_{i j}^{2} / c_{i} c_{j}$, where $c_{i}$ stands for the number of documents that include $i$, and $c_{i j}$ accounts for the number of documents that contain both $i$ and $j$. Then, the topics were identified with the simple center clustering algorithm [21], which searches for highly tied keywords according to their $e_{i j}$. Thus each topic corresponds to a group of keywords, i.e., to a thematic network.

The role of a thematic network is characterized in function of two measures [21]:

- Centrality calculates the degree of interaction of a thematic network with the remaining ones as $c=$ $10 \sum e_{i o}$, where $i$ and $o$ are keywords inside and outside the network.

\footnotetext{
${ }^{1}$ https://sci2s.ugr.es/scimat/
} 
- Density characterizes the network internal consistency as $d=100 \sum e_{i j} / w$, where $i$ and $j$ are every pair of keywords inside the network, and $w$ is the total number of keywords the network has.

Strategic diagrams help to visualize both centrality and density distributions. To do so, the centrality and density normalized rankings are calculated as $\operatorname{rank}\left(c_{i}\right) / n$ and $\operatorname{rank}\left(d_{i}\right) / n$, where $\operatorname{rank}\left(c_{i}\right)$ and $\operatorname{rank}\left(d_{i}\right)$ are the positions of the theme $i$ in the centrality and density rankings sorted in ascending order, respectively. Notice that $\operatorname{rank}\left(c_{i}\right)$ and $\operatorname{rank}\left(d_{i}\right)$ are divided by the total number of themes $n$ to normalize their values into the interval $[0,1]$. The strategic diagram $x$-axis and $y$-axis account for the centrality and density normalized rankings, respectively. Figure 2 shows the possible roles a thematic network may have according to the strategic diagram quadrant where it is placed.

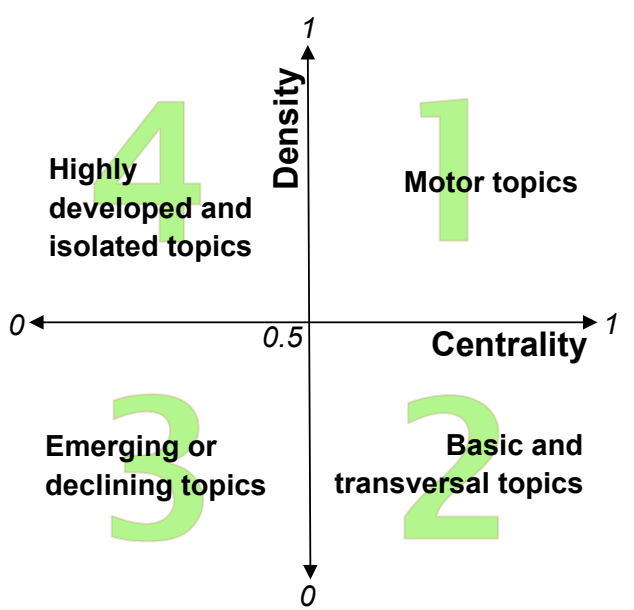

Figure 2: Strategic diagram quadrants.

\subsection{Material}

In accordance with open science's good practices, the data we have analyzed, together with a detailed report of the results, are publicly available at:

https://github.com/rheradio/ML-SE-BibAnalysis

\section{Results and discussion}

This section reports the most significant results of our article bibliometric corpus analysis.

\subsection{How fast is the number of publications growing? (RQ1)}

Figure 3 shows the distribution of the analyzed paper sample over time. In the period this article is focused,
2015-2019, there has been an explosion of interest in applying machine learning techniques to tackle software engineering problems.

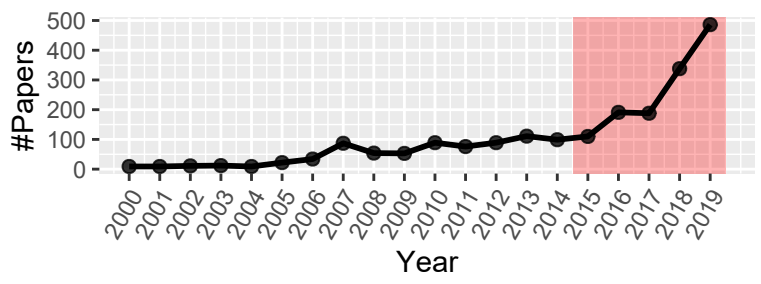

Figure 3: Number of publications per year.

\subsection{Who are the most productive authors, and how do they collaborate? (RQ2)}

A total of 2,862 researchers have co-authored the 1,312 papers that this article analyzes. Most of them are causal authors, as $75.99 \%$ have published a single paper, and only $3.33 \%$ have published five or more papers. This is consistent with one of the fundamental laws in bibliometrics, called Lotka's law [22], which states that the number of authors with $n$ papers is usually inversely proportional to $n^{2}$. In our case, 2175 authors have written one article, so Lotka's law predicts that the number of authors that have published $n$ papers should be $\frac{2175}{n^{2}}$. Figure 4 compares the empirical distribution found in the sample with the distribution predicted by Lotka's law.

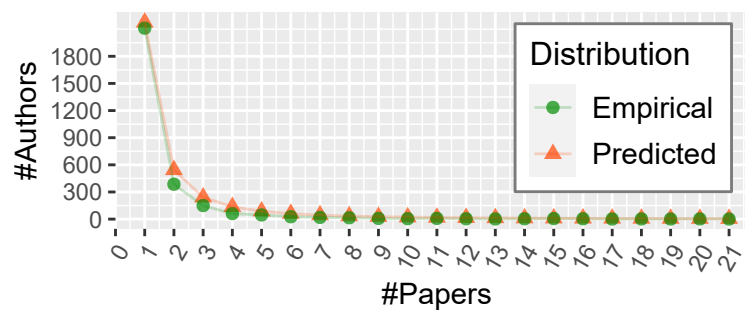

Figure 4: Authorship distribution fits Lotka's law.

Figure 5 shows the collaboration networks among the most prolific researchers that have published at least five papers. There is an edge between two researchers whenever they have co-authored some paper. Node sizes and edge widths are proportional to the number of published articles and the equivalence indices between authors, respectively. The graph is colored according to the clusters of collaborating authors identified with the Leiden algorithm [23]. 


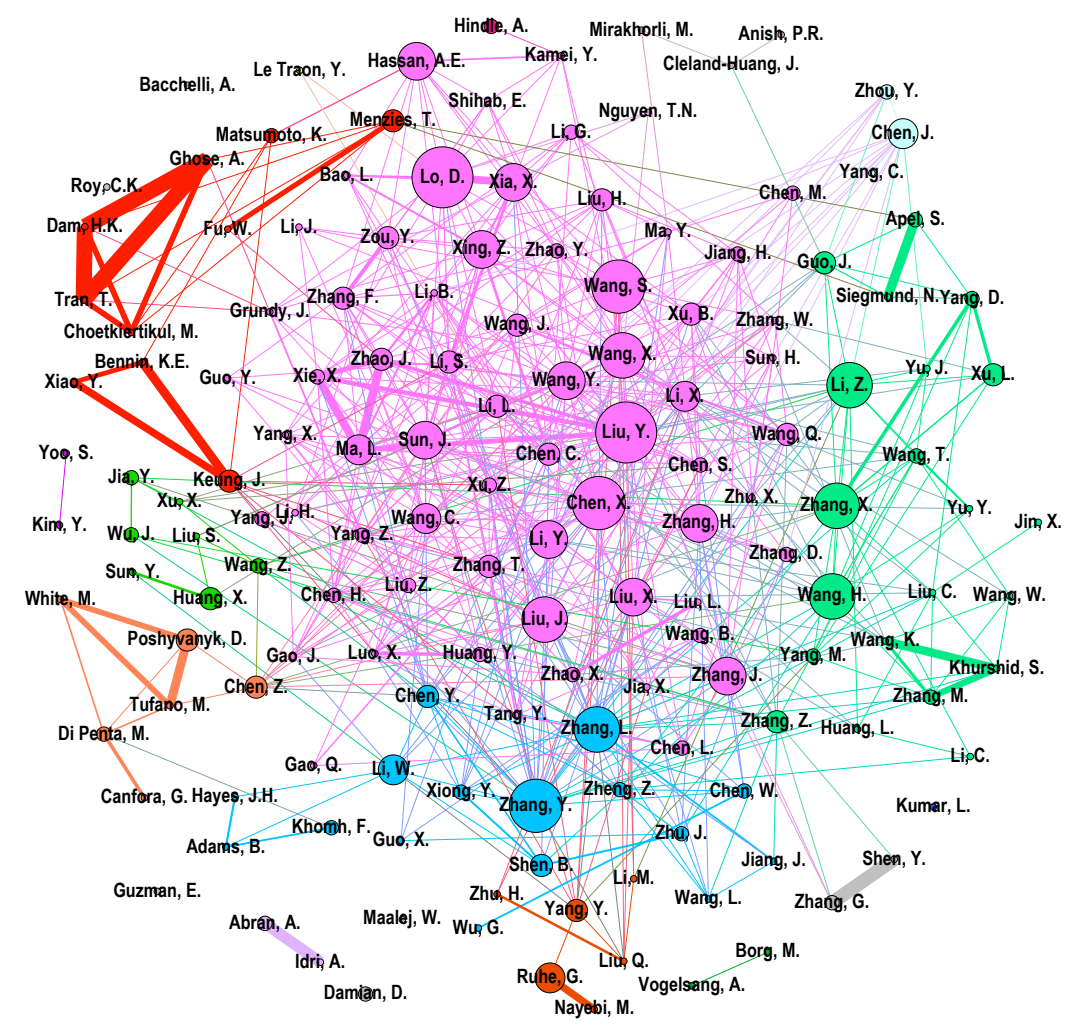

Figure 5: Collaboration networks of the authors that have published at least five papers.

\subsection{What countries and institutions are leading research? (RQ3)}

Figure 6 highlights the countries that have contributed with the most papers: China $(27.81 \%$ of all published papers), USA (15.23\%), Canada (7\%), and Germany $(4.17 \%)$. Figure 7 summarizes the institutions whose researchers have published the greatest number of papers.

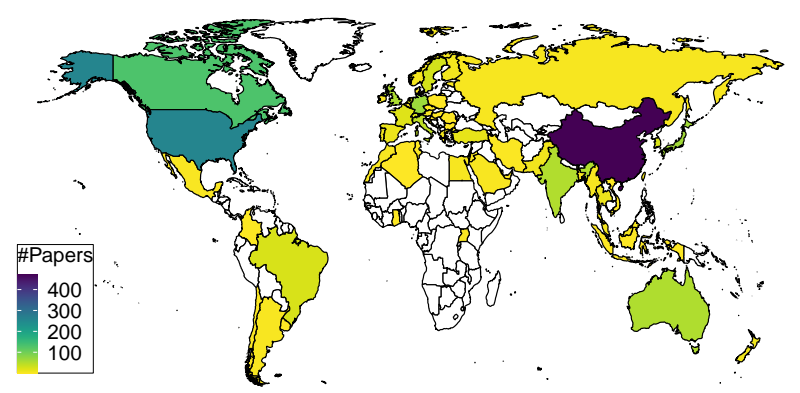

Figure 6: Most prolific countries.

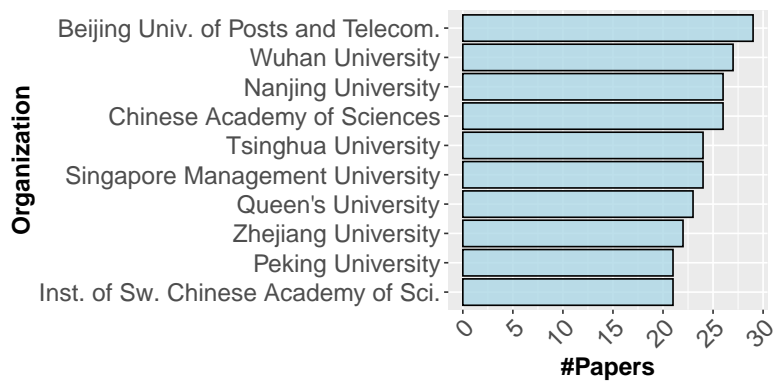

Figure 7: Most prolific institutions.

\subsection{What journals have published the most articles? (RQ4)}

Figure 8 shows the journals that have published the highest number of papers. 


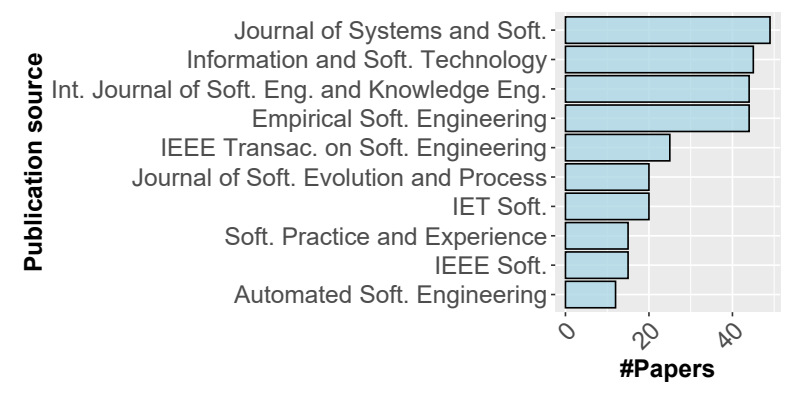

Figure 8: Most prolific journals.

\subsection{What are the most relevant themes of research, and what are the highest impacted papers for those themes? (RQ5)}

Our science mapping analysis reveals five thematic networks, whose role is represented in the strategic diagram in Figure 9 (the size of each network is proportional to its number of associated articles). There are two motor topics (classification and defect prediction), one traversal topic (genetic algorithms), one emerging topic (topic modeling), and one developed but isolated topic (security).

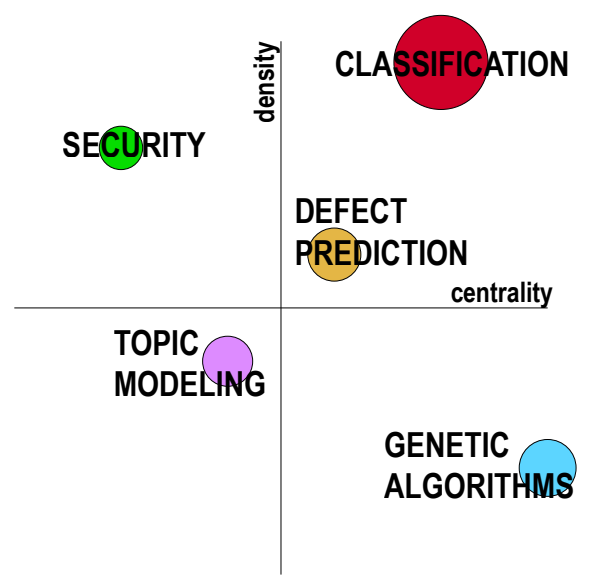

Figure 9: Strategic diagram.

Figures 10-14 detail each thematic network. Node sizes reflect the number of papers that include the corresponding keywords. There is an edge connecting two keywords whenever they co-occur in some paper. The edge width is proportional to the keywords equivalence index.

Table 1 summarizes the papers that have received the highest number of citations in each thematic network. The last column follows the notation [reference]\#citations, e.g., [24] $]_{132}$ means that [24] has been cited 132 times since its publication in 2015. It is worth noting that some papers deal with different topics, and thus they appear several times in the table (e.g., [24] encompasses classification and defect prediction). A more exhaustive list of articles per topic is available at this paper public repository (see Section 2.3).

Table 1: H-index and highest impacted papers per thematic network.

\begin{tabular}{|c|c|c|}
\hline Thematic network & H-index & Top-10 Papers \\
\hline CLASSIFICATION & 29 & $\begin{array}{l}{[24]_{132},[25]_{131},} \\
{[26]_{128},[27]_{116}} \\
{[28]_{97},[29]_{73},} \\
{[8]_{61},[30]_{61},} \\
{[31]_{58},[32]_{54},} \\
{[33]_{53},[34]_{51}}\end{array}$ \\
\hline DEFECT-PREDICTION & 24 & $\begin{array}{l}{[24]_{132},[27]_{116},} \\
{[35]_{115},[36]_{70}} \\
{[37]_{65},[38]_{58}} \\
{[39]_{51},[40]_{45}} \\
{[41]_{43},[42]_{40}}\end{array}$ \\
\hline GENETIC-ALGORITHMS & 21 & $\begin{array}{l}{[26]_{128},[43]_{79},} \\
{[29]_{73},[9]_{55}} \\
{[44]_{45},[45]_{43}} \\
{[46]_{43},[47]_{39}} \\
{[48]_{35},[49]_{34}}\end{array}$ \\
\hline TOPIC-MODELING & 21 & $\begin{array}{l}{[25]_{131},[50]_{83},} \\
{[29]_{73},[51]_{62}} \\
{[38]_{58},[34]_{51}} \\
{[52]_{48},[53]_{48}} \\
{[54]_{44},[55]_{43}}\end{array}$ \\
\hline SECURITY & 10 & $\begin{array}{l}{[31]_{58},[45]_{43},} \\
{[48]_{35},[56]_{30}} \\
{[57]_{28},[58]_{26}} \\
{[59]_{20},[60]_{16}} \\
{[61]_{13},[62]_{11}}\end{array}$ \\
\hline
\end{tabular}

3.5.1. Thematic Network 1: Classification (Figure 10). $44.28 \%$ of the papers in the sample deal with classification, thus being the most common ML application in the software engineering field. For example, ML approaches are used (i) to distinguish between potentially defective and correct software components [24, 27, 29, 52], (ii) to identify code clones, caused by copying \& pasting code fragments instead of using software abstractions that favor maintenance [25], (iii) to classify software application reviews [8, 53], (iv) to distinguish relevant knowledge published on forums about software development (e.g., Stack Overflow) [30], (v) to detect if a given code fragment is vulnerable from a security point of view [31], (vi) to extract traceability links between software artifacts coming from different life-cycle stages [32], (vii) to classify requirements into functional and non-functional [63], etc.

For that, different techniques are used, e.g., support vector machines (SVMs) [24], neural networks [25, 26, 
28, 29, 30, 32], natural language processing (NLP) [29, 8,30 ], $k$ nearest neighbors (k-NN) [52], naive Bayes [63], feature selection [64], etc.

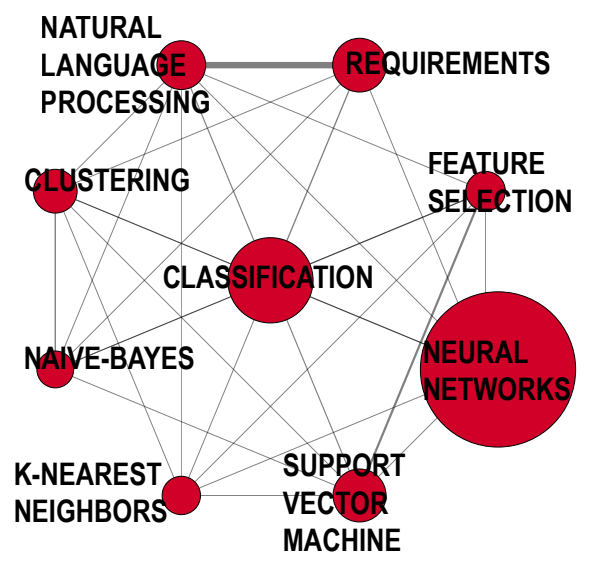

Figure 10: Thematic network CLASSIFICATION.

3.5.2. Thematic Network 2: Defect Prediction (Figure 11). As software complexity increases, more rigorous quality control procedures are required to guarantee application correctness. However, the scarcity of human and financial resources makes it desirable to automate quality control as much as possible. Accordingly, much research analyzes existing projects to learn the symptoms that will help to identify error-prone components in future projects $[24,27,35$, $36,37,38,39]$, or artifacts that will require a major development and maintenance effort $[39,65,66]$.

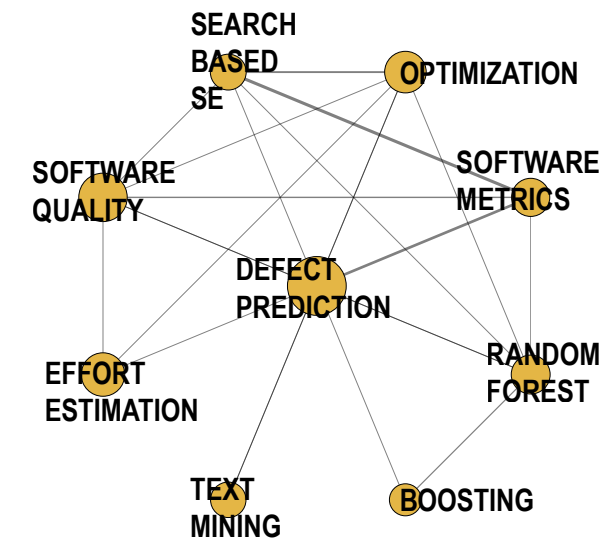

Figure 11: Thematic network DEFECT-PREDICTION.
3.5.3. Thematic Network 3: Genetic Algorithms (Figure 12). A bio-inspired technique, known as mutation testing, tries to assess the capacity of a test suite to detect errors by slightly modifying a program [67, 68]. Other bio-inspired approaches look for automatizing fault debugging by learning how to rank software components according to their likelihood of being the cause of a given problem [49]. Also, some genetic programming approaches try to sort bugs according to their severity by examining bug reports [46].

Other ML techniques are used to target different issues, such as (i) refactoring code to fix design problems, improving this way software quality and maintenance [43, 69], or (ii) predicting the future behavior of cloud systems to adjust the resources they may need in advance [47].
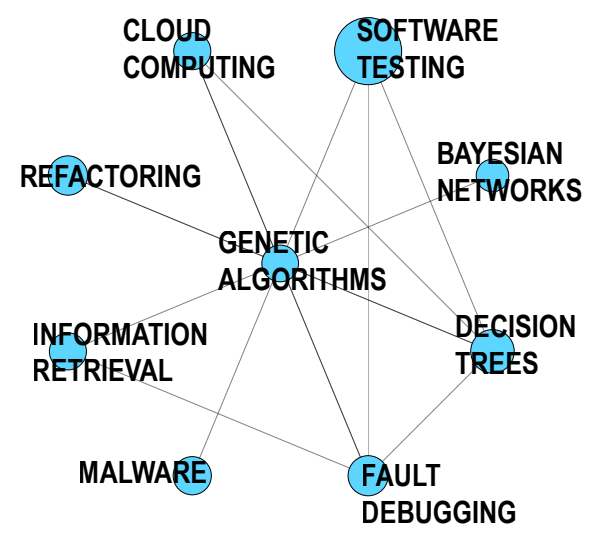

Figure 12: Thematic net. GENETIC-ALGORITHMS.

3.5.4. Thematic Network 4: Topic Modeling (Figure 13). NLP techniques, such as topic modeling, typically Latent Dirichlet Allocation (LDA), and sentiment analysis, are used for various purposes: (i) to extract information from developers' discussion forums to complement and improve API documentation [50, 51], (ii) to classify application reviews [53], (iii) to assign bugs to the most convenient developers for fixing them [55], (iv) to classify, cluster and rank tweets about software applications [70, 71], (v) to adjust software maintenance by classifying changes into corrective and adaptive [72], etc. 


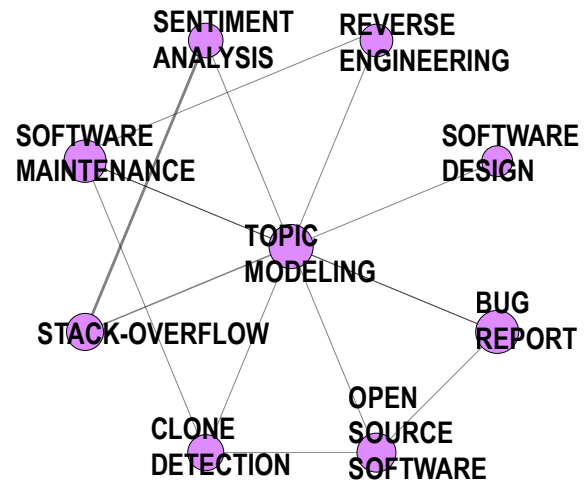

Figure 13: Thematic network TOPIC-MODELING.

3.5.5. Thematic Network 5: Security (Figure 14). Another important ML application field is software security. For instance, to repeatedly test an application with modified inputs for finding vulnerabilities [31, 60], to detect malware [45], to recognize run-time system anomalies [59], to complement firewalls by detecting network intrusions [62], etc. It is worth noting that many of these applications are validated on the Android operating system $[45,48]$.

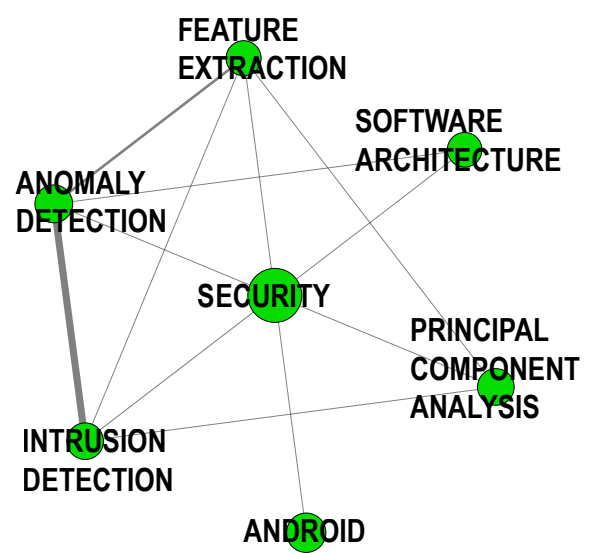

Figure 14: Thematic network SECURITY.

\section{Conclusions}

In the last five years, there has been an explosion of articles about ML for software engineering, being mostly published by Chinese and American researchers.

Although ML is applied to a variety of software engineering problems, most literature is focused on software testing and security, including fault/vulnerability detection, localization, and prediction. To do so, neural networks are typically used, particularly deep neural networks. Other common techniques are SVMs, decision trees, naive Bayes, k-NN, Bayesian networks, and genetic algorithms.

As an emerging line of research, NLP and text mining techniques, such as topic modeling (e.g., LDA) and sentiment analysis, have began to be used for analyzing developers forums (e.g., Stack Overflow), application reviews, commits and issues from version control systems (e.g., GitHub), etc. Then, the extracted knowledge is applied to handle different questions, such as supporting software maintenance, improving API documentation, predicting defects, estimating software cost, etc.

The reduced number of articles reporting the use of other popular ML techniques, such as regression methods (e.g., multivariate linear regression, logistic regression, regression trees, and model trees), or rule induction algorithms (e.g., decision rules and association rules) is striking. Perhaps this points a direction for future research. Anyway, we believe ML can contribute to many other software engineering areas, such as requirement analysis, design validation, code generation, etc. Consequently, we think that this research field will keep growing in the future.

Our study provides a general perspective obtained with the automated analysis of an extensive article corpus. This overview might be complemented with more in-depth reviews focused on more specific topics, such as software failure prediction, artifact traceability, support for highly configurable software systems, etc.

\section{Acknowledgements}

This work has been supported by (i) the Spanish Ministry of Science, Innovation and Universities, under grants with reference DPI2016-77677-P and PID2019-105381GA-I00 (iScience), and (ii) the Community of Madrid, under the research network CAM RoboCity2030 S2013/MIT-2748.

\section{References}

[1] K. Elish and M. Elish, "Predicting defect-prone software modules using support vector machines," Journal of Systems and Software, vol. 81, no. 5, pp. 649-660, 2008.

[2] E. Arisholm, L. Briand, and E. Johannessen, "A systematic and comprehensive investigation of methods to build and evaluate fault prediction models," Journal of Systems and Software, vol. 83, no. 1, pp. 2-17, 2010.

[3] Y. Ma, G. Luo, X. Zeng, and A. Chen, "Transfer learning for cross-company software defect prediction," Information and Software Technology, vol. 54, no. 3, pp. 248-256, 2012.

[4] S. Wang, T. Liu, and L. Tan, "Automatically learning semantic features for defect prediction," in International 
Conference on Software Engineering (ICSE), (Austin, Texas, USA), 2016.

[5] J. Wen, S. Li, Z. Lin, Y. Hu, and C. Huang, "Systematic literature review of machine learning based software development effort estimation models," Information and Software Technology, vol. 54, no. 1, pp. 41-59, 2012.

[6] Y. Li, M. Xie, and T. Goh, "A study of project selection and feature weighting for analogy based software cost estimation," Journal of Systems and Software, vol. 82, no. 2, pp. 241-252, 2009.

[7] M. Azeem, F. Palomba, L. Shi, and Q. Wang, "Machine learning techniques for code smell detection: A systematic literature review and meta-analysis," Information and Software Technology, vol. 108, pp. 115-138, 2019.

[8] W. Maalej, Z. Kurtanović, H. Nabil, and C. Stanik, "On the automatic classification of app reviews," Requirements Engineering, vol. 21, no. 3, pp. 311-331, 2016.

[9] M. Alazab, "Profiling and classifying the behavior of malicious codes," Journal of Systems and Software, vol. 100, pp. 91-102, 2015.

[10] M. J. Cobo, A. Lopez-Herrera, E. Herrera-Viedma, and F. Herrera, "Science mapping software tools: Review, analysis, and cooperative study among tools," Journal of the American Society for Information Science and Technology, vol. 62, no. 7, pp. 1382-1402, 2011.

[11] R. Heradio, D. Fernandez-Amoros, J. A. Moral-Muñoz, and M. J. Cobo, "Rough Sets: A Bibliometric Analysis from 2014 to 2018," in Hawaii International Conference on System Sciences, HICSS, (Maui, Hawaii), 2020.

[12] E. Noyons, H. Moed, and A. Van Raan, "Integrating research performance analysis and science mapping," Scientometrics, vol. 46, no. 3, pp. 591-604, 1999.

[13] M. J. Cobo, M. A. Martínez, M. Gutiérrez-Salcedo, H. Fujita, and E. Herrera-Viedma, " 25 years at Knowledge-Based Systems: A bibliometric analysis," Knowledge-Based Systems, vol. 80, pp. 3-13, 2015.

[14] R. Heradio, H. Perez-Morago, D. Fernandez-Amoros, F. J. Cabrerizo, and E. Herrera-Viedma, "A bibliometric analysis of 20 years of research on software product lines," Information and Software Technology, vol. 72, pp. 1 - 15, 2016.

[15] R. Heradio, D. Fernandez-Amoros, C. Cerrada, and M. J. Cobo, "Group Decision-Making Based on Artificial Intelligence: A Bibliometric Analysis," Mathematics, vol. 8, no. 9, pp. 1-20, 2020.

[16] D. Moher, A. Liberati, J. Tetzlaff, and D. Altman, "Preferred reporting items for systematic reviews and meta-analyses: the PRISMA statement," PLoS Medicine, vol. 6, no. 7, pp. 1-6, 2009.

[17] K. Börner, C. Chen, and K. W. Boyack, "Visualizing knowledge domains," Annual Review of Information Science and Technology, vol. 37, no. 1, pp. 179-255, 2003.

[18] B. Kitchenham and S. Charters, "Guidelines for performing systematic literature reviews in software engineering," tech. rep., Keele University and University of Durham, UK, 2007.

[19] C. Wohlin, P. Runeson, P. A. da Mota Silveira Neto, E. Engström, I. do Carmo Machado, and E. S. de Almeida, "On the reliability of mapping studies in software engineering," Journal of Systems and Software, vol. 86, no. 10, pp. $2594-2610,2013$.
[20] E. Aranda-Escolástico, M. Guinaldo, R. Heradio, J. Chacon, H. Vargas, J. Sánchez, and S. Dormido, "Event-based control: A bibliometric analysis of twenty years of research," IEEE Access, vol. 8, pp. 47188-47208, 2020.

[21] M. Callon, J. Courtial, and F. Laville, "Co-word analysis as a tool for describing the network of interactions between basic and technological research: The case of polymer chemsitry," Scientometrics, no. 1, pp. 155-205, 1991.

[22] A. J. Lotka, "The frequency distribution of scientific productivity," Journal of the Washington academy of sciences, vol. 16, no. 12, pp. 317-323, 1926.

[23] V. A. Traag, L. Waltman, and N. J. van Eck, "From Louvain to Leiden: guaranteeing well-connected communities," Nature. Scientific Reports, vol. 9, no. 1, pp. 1-12, 2019.

[24] I. Laradji, M. Alshayeb, and L. Ghouti, "Software defect prediction using ensemble learning on selected features," Information and Software Technology, vol. 58, pp. 388-402, 2015.

[25] M. White, M. Tufano, C. Vendome, and D. Poshyvanyk, "Deep learning code fragments for code clone detection," in International Conference on Automated Software Engineering (ASE), (Singapore), 2016.

[26] S. Jana, Y. Tian, K. Pei, and B. Ray, "Deeptest: Automated testing of deep-neural-network-driven autonomous cars," in International Conference on Software Engineering (ICSE), (Gothenburg, Sweden), 2018.

[27] C. Tantithamthavorn, S. McIntosh, A. Hassan, and K. Matsumoto, "Automated parameter optimization of classification techniques for defect prediction models," in International Conference on Software Engineering (ICSE), (Austin, Texas, USA), 2016.

[28] M. White, C. Vendome, M. Linares-Vásquez, and D. Poshyvanyk, "Toward deep learning software repositories," in International Working Conference on Mining Software Repositories (MSR), (Florence, Italy), 2015.

[29] A. Lam, A. Nguyen, H. Nguyen, and T. Nguyen, "Combining deep learning with information retrieval to localize buggy files for bug reports," in International Conference on Automated Software Engineering (ASE), (Lincoln, NE, USA), 2015.

[30] B. Xu, D. Ye, Z. Xing, X. Xia, G. Chen, and $\mathrm{S}$. Li, "Predicting semantically linkable knowledge in developer online forums via convolutional neural network," in International Conference on Automated Software Engineering (ASE), (Singapore), 2016.

[31] P. Godefroid, H. Peleg, and R. Singh, "Learn\&fuzz: Machine learning for input fuzzing," in International Conference on Automated Software Engineering (ASE), (Urbana, IL, USA), 2017.

[32] J. Guo, J. Cheng, and J. Cleland-Huang, "Semantically enhanced software traceability using deep learning techniques," in International Conference on Software Engineering (ICSE), (Buenos Aires, Argentina), 2017.

[33] X. Gu, H. Zhang, and S. Kim, "Deep code search," in International Conference on Software Engineering (ICSE), (Gothenburg, Sweden), 2018.

[34] P. Louridas and C. Ebert, "Machine learning," IEEE Software, vol. 33, no. 5, pp. 110-115, 2016. 
[35] P. He, B. Li, X. Liu, J. Chen, and Y. Ma, "An empirical study on software defect prediction with a simplified metric set," Information and Software Technology, vol. 59, pp. 170-190, 2015.

[36] L. Chen, B. Fang, Z. Shang, and Y. Tang, "Negative samples reduction in cross-company software defects prediction," Information and Software Technology, vol. 62, no. 1, pp. 67-77, 2015.

[37] D. Ryu, O. Choi, and J. Baik, "Value-cognitive boosting with a support vector machine for cross-project defect prediction," Empirical Software Engineering, vol. 21, no. 1, pp. 43-71, 2016.

[38] W. Fu, T. Menzies, and X. Shen, "Tuning for software analytics: Is it really necessary?," Information and Software Technology, vol. 76, pp. 135-146, 2016.

[39] X. Yang, D. Lo, X. Xia, and J. Sun, "Tlel: A two-layer ensemble learning approach for just-in-time defect prediction," Information and Software Technology, vol. 87, pp. 206-220, 2017.

[40] J. Huang, Y.-F. Li, and M. Xie, "An empirical analysis of data preprocessing for machine learning-based software cost estimation," Information and Software Technology, vol. 67, pp. 108-127, 2015.

[41] A. Idri, M. Hosni, and A. Abran, "Systematic literature review of ensemble effort estimation," Journal of Systems and Software, vol. 118, pp. 151-175, 2016.

[42] H. Han, X. Guo, and H. Yu, "Variable selection using mean decrease accuracy and mean decrease gini based on random forest," in International Conference on Software Engineering and Service Sciences (ICSESS), (Beijing,China), 2016.

[43] F. Arcelli Fontana, M. Mäntylä, M. Zanoni, and A. Marino, "Comparing and experimenting machine learning techniques for code smell detection," Empirical Software Engineering, vol. 21, no. 3, pp. 1143-1191, 2016.

[44] L. Ma, F. Juefei-Xu, F. Zhang, J. Sun, M. Xue, B. Li, C. Chen, T. Su, L. Li, Y. Liu, J. Zhao, and Y. Wang, "Deepgauge: Multi-granularity testing criteria for deep learning systems," in International Conference on Automated Software Engineering (ASE), (Montpellier, France), 2018.

[45] S. Wu, P. Wang, X. Li, and Y. Zhang, "Effective detection of android malware based on the usage of data flow apis and machine learning," Information and Software Technology, vol. 75, pp. 17-25, 2016.

[46] S. Guo, R. Chen, H. Li, T. Zhang, and Y. Liu, "Identify Severity Bug Report with Distribution Imbalance by CR-SMOTE and ELM," International Journal of Software Engineering and Knowledge Engineering, vol. 29, no. 2, pp. 139-175, 2019.

[47] A. Nikravesh, S. Ajila, and C.-H. Lung, "Towards an autonomic auto-scaling prediction system for cloud resource provisioning," in International Symposium on Software Engineering for Adaptive and Self-Managing Systems (SEAMS), (Firenze, Italy), 2015.

[48] K. Allix, T. Bissyandé, Q. Jérome, J. Klein, R. State, and Y. Le Traon, "Empirical assessment of machine learning-based malware detectors for android: Measuring the gap between in-the-lab and in-the-wild validation scenarios," Empirical Software Engineering, vol. 21, no. 1, pp. 183-211, 2016.
[49] J. Sohn and S. Yoo, "FLUCCS: Using code and change metrics to improve fault localization," in International Symposium on Software Testing and Analysis (ISSTA), (Santa Barbara, CA, United States), 2017.

[50] C. Treude and M. Robillard, "Augmenting API documentation with insights from stack overflow," in International Conference on Software Engineering (ICSE), (Austin, Texas, USA), 2016.

[51] C. Rosen and E. Shihab, "What are mobile developers asking about? a large scale study using stack overflow," Empirical Software Engineering, vol. 21, no. 3, pp. 1192-1223, 2016.

[52] T. Zhang, J. Chen, G. Yang, B. Lee, and X. Luo, "Towards more accurate severity prediction and fixer recommendation of software bugs," Journal of Systems and Software, vol. 117, pp. 166-184, 2016.

[53] E. Guzman, M. El-Haliby, and B. Bruegge, "Ensemble methods for app review classification: An approach for software evolution," in International Conference on Automated Software Engineering (ASE), (Lincoln, NE, USA), 2015.

[54] N. Walkinshaw, R. Taylor, and J. Derrick, "Inferring extended finite state machine models from software executions," Empirical Software Engineering, vol. 21, no. 3, pp. 811-853, 2016.

[55] X. Xia, D. Lo, Y. Ding, J. Al-Kofahi, T. Nguyen, and X. Wang, "Improving automated bug triaging with specialized topic model," IEEE Transactions on Software Engineering, vol. 43, no. 3, pp. 272-297, 2017.

[56] C. Sauvanaud, K. Lazri, M. Kaaniche, and K. Kanoun, "Anomaly detection and root cause localization in virtual network functions," in International Symposium on Software Reliability Engineering (ISSRE), (Ottawa, Canada), 2016.

[57] M. Mirakhorli and J. Cleland-Huang, "Detecting, tracing, and monitoring architectural tactics in code," IEEE Transactions on Software Engineering, vol. 42, no. 3, pp. 206-221, 2016.

[58] C. Bertero, M. Roy, C. Sauvanaud, and G. Tredan, "Experience report: Log mining using natural language processing and application to anomaly detection," in International Symposium on Software Reliability Engineering (ISSRE), (Toulouse, France), 2017.

[59] W. Khreich, B. Khosravifar, A. Hamou-Lhadj, and C. Talhi, "An anomaly detection system based on variable n-gram features and one-class svm," Information and Software Technology, vol. 91, pp. 186-197, 2017.

[60] I. Medeiros, N. Neves, and M. Correia, "DEKANT: A static analysis tool that learns to detect web application vulnerabilities," in International Symposium on Software Testing and Analysis (ISSTA), (Saarbrücken, Germany), 2016.

[61] J. Zhu, S. He, J. Liu, P. He, Q. Xie, Z. Zheng, and M. Lyu, "Tools and benchmarks for automated log parsing," in International Conference on Software Engineering: Software Engineering in Practice (ICSE-SEIP), (Montreal, QC, Canada), 2019.

[62] Y. Aung and M. Min, "An analysis of random forest algorithm based network intrusion detection system," in International Conference on Software Engineering, Artificial Intelligence, Networking and Parallel/Distributed Computing (SNPD), (Kanazawa, Japan), 2017. 
[63] Z. Abad, O. Karras, P. Ghazi, M. Glinz, G. Ruhe, and K. Schneider, "What works better? a study of classifying requirements," in International Requirements Engineering Conference (RE), (Lisbon, Portugal), 2017.

[64] L. Kumar, S. Sripada, A. Sureka, and S. Rath, "Effective fault prediction model developed using Least Square Support Vector Machine (LSSVM)," Journal of Systems and Software, vol. 137, pp. 686-712, 2018.

[65] X. Chen, Y. Zhao, Q. Wang, and Z. Yuan, "Multi: Multi-objective effort-aware just-in-time software defect prediction," Information and Software Technology, vol. 93, pp. 1-13, 2018.

[66] J. Moeyersoms, E. Junqué De Fortuny, K. Dejaeger, B. Baesens, and D. Martens, "Comprehensible software fault and effort prediction: A data mining approach," Journal of Systems and Software, vol. 100, pp. 80-90, 2015.

[67] J. Zhang, Z. Wang, L. Zhang, D. Hao, L. Zang, S. Cheng, and L. Zhang, "Predictive mutation testing," in International Symposium on Software Testing and Analysis (ISSTA), (Saarbrücken, Germany), 2016.

[68] J. Wang, G. Dong, J. Sun, X. Wang, and P. Zhang, "Adversarial sample detection for deep neural network through model mutation testing," in International Conference on Software Engineering (ICSE), (Montreal, Canada), 2019.

[69] T. Sharma and D. Spinellis, "A survey on software smells," Journal of Systems and Software, vol. 138, pp. 158-173, 2018.

[70] E. Guzman, M. Ibrahim, and M. Glinz, "A little bird told me: Mining tweets for requirements and software evolution," pp. 11-20, 2017.

[71] E. Guzman, R. Alkadhi, and N. Seyff, "An exploratory, study of twitter messages about software applications," Requirements Engineering, vol. 22, no. 3, pp. 387-412, 2017.

[72] Y. Fu, M. Yan, X. Zhang, L. Xu, D. Yang, and J. Kymer, "Automated classification of software change messages by semi-supervised latent dirichlet allocation," Information and Software Technology, vol. 57, no. 1, pp. 369-377, 2015. 